\title{
GROUPS WITH COMMUTING POWERS
}

\author{
ROLF BRANDL
}

(Received 28 July 2008)

\begin{abstract}
A group $G$ satisfies the second Engel condition $[X, Y, Y]=1$ if and only if $x$ commutes with $x^{y}$, for all $x, y \in G$. This paper considers the generalization of this condition to groups $G$ such that, for fixed positive integers $r$ and $s, x^{r}$ commutes with $\left(x^{s}\right)^{y}$ for all $x, y \in G$. Various general bounds are proved for the structure of groups in the corresponding variety, defined by the law $\left[X^{r},\left(X^{s}\right)^{Y}\right]=1$.
\end{abstract}

2000 Mathematics subject classification: primary 20F19, 20F12, 20F45; secondary 20E10, 20D60, 20D10.

Keywords and phrases: two-Engel, Bell groups, variety of groups.

\section{Introduction}

Let $G$ be a group. Suppose that in every conjugacy class of $G$, every two elements commute. Hence $\left[x, x^{y}\right]=1$ for all $x, y \in G$. This clearly is equivalent so saying then every element $x \in G$ generates an Abelian normal subgroup of $G$, which in turn is equivalent to the second Engel Condition $[y, x, x]=1$ for all $x, y \in G$. It is known that groups satisfying one of these equivalent conditions is nilpotent of class at most three.

The above notion has been generalized in various directions. For example, every two-Engel group for every integer $n$ satisfies the following conditions discussed in [7] and [2]:

$$
\begin{array}{ll}
{\left[x^{n}, y\right]} & =[x, y]^{n} \quad(n \text {-Levi property }), \\
{\left[x^{n}, y\right]} & =\left[x, y^{n}\right] \quad(n \text {-Bell property }) .
\end{array}
$$

In this paper, we consider another generalization of two-Engel groups. Clearly, if $x$ and $x^{y}$ commute, then so do all powers $x^{r}$ and $\left(x^{y}\right)^{s}$ where $r, s$ are integers.

For positive integers $r, s$, let

$$
\mathcal{O}(r, s)=\left\{G \mid G \text { satisfies the law }\left[X^{r},\left(X^{s}\right)^{Y}\right]=1\right\}
$$

(C) 2009 Australian Mathematical Society 0004-9727/2009\$16.00 
denote the class of all groups with this property. This class clearly is a variety defined by one single law.

In the second section, we present typical examples for groups in $\mathcal{O}(r, s)$ for various values of $r$ and $s$, while the third one contains various general bounds for the structure of groups in $\mathcal{O}(r, s)$. In the final section, structural results on finite groups in $\mathcal{O}(r, s)$ are proved in case where the defining parameters $r$ and $s$ are 'small' in some sense.

We use standard notation throughout. All commutators are left-normed. In addition, by $o(x)$ we denote the order of the element $x$, and $G=[N] Q$ indicates that the group $G$ is a split extension of a normal subgroup $N$ of $G$ by a complement $Q$. The class of all Abelian groups will be denoted by $\mathfrak{A}$, and the class of all finite nilpotent groups by $\mathcal{N}$.

\section{Examples}

Assume that the order of every element $x \in G$ either divides $r$ or $s$ or maybe both (where $r$ and $s$ are fixed positive integers). Then we have the disjunction $x^{r}=1$ OR $x^{s}=1$ for all elements $x \in G$. In particular, $G \in \mathcal{O}(r, s)$. This idea was used in [3] to determine a basis for the laws of of $\operatorname{PSL}(2,5)$.

Clearly, $\mathcal{O}(r, s)$ contains all groups of exponent dividing $r$ or $s$. Moreover, it contains all Abelian groups.

If $x^{r}$ commutes with all conjugates of $x^{s}$, then conversely, all conjugates of $x^{r}$ commute with $x^{s}$. Thus $\mathcal{O}(r, s)=\mathcal{O}(s, r)$ for all $r, s$.

EXAMPLE 1. $\mathcal{O}(1,1)$ is the class of all groups with the law $\left[X, X^{Y}\right]=1$, thus $\mathcal{O}(1,1)$ is the class of all groups satisfying the second Engel condition.

If $G$ is second Engel, then for all $x, y \in G$ we have that $x^{r}$ commutes with $\left(x^{s}\right)^{y}$, and so $G \in \mathcal{O}(r, s)$. Hence $\mathcal{O}(r, s)$ contains all second Engel groups. Thus, the classes $\mathcal{O}(r, s)$ generalize the second Engel groups.

EXAMPLE 2. We have $\mathfrak{A} \mathfrak{A}_{r} \subseteq \mathcal{O}(r, s)$ and $\mathfrak{A} \mathfrak{A}_{s} \subseteq \mathcal{O}(r, s)$ for all $r, s$.

Proof. Let $G \in \mathfrak{A A}_{r}$, and let $N$ be an Abelian normal subgroup of $G$ such that $G / N$ is Abelian of exponent dividing $r$. For all $x, y \in G$,

$$
\left[x^{r},\left(x^{s}\right)^{y}\right]=\left[x^{r}, x^{s}\left[x^{s}, y\right]\right]=\left[x^{r},\left[x^{s}, y\right]\right] .
$$

As $x^{r} \in N$ and $\left[x^{s}, y\right] \in G^{\prime} \leq N$ and $N$ is Abelian, we get $\left[x^{r},\left(x^{s}\right)^{y}\right]=1$. This shows $G \in \mathcal{O}(r, s)$. The second claim follows in the same way.

We now consider another typical example in which one can read off the exponents $r$ and $s$ from the group.

ExAmple 3. Let $G=[N] Q$ be a Frobenius group, and set $r=\exp (N)$ and $s=$ $\exp (Q)$. As every element of $G \backslash N$ is contained in some conjugate of $Q$, we see that $G \in \mathcal{O}(r, s)$. If $N$ is Abelian, then $G \in \mathcal{O}(1, s)$. Indeed, if $x \in N$, then $x^{y} \in N$, and so $\left[x, x^{y}\right]=1$. If $x \in G \backslash N$, then $x^{s}=1$. 


\section{Some general bounds}

We first collect some absolutely basic properties of groups in $\mathcal{O}(r, s)$.

Lemma 4. Let $G$ be a group in $\mathcal{O}(r, s)$. Then we have the following results.

(a) For every element $x \in G$, we have $x^{r} \in C_{G}\left(\left\langle x^{s}\right\rangle^{G}\right)$ and $x^{s} \in C_{G}\left(\left\langle x^{r}\right\rangle^{G}\right)$.

(b) For all $x, y \in G$, we have $\left[y, x^{s}, x^{r}\right]=1$.

(c) For all positive integers $\lambda, \mu$, we have $G \in \mathcal{O}(\lambda r, \mu s)$.

(d) Let $n=\operatorname{lcm}(r, s)$. For all $x, y \in G$, we have $\left[y, x^{n}, x^{n}\right]=1$ and $\left[x^{n},\left(x^{n}\right)^{y}\right]=1$.

(e) If $o(x)$ is finite and coprime to $r s$, then $\left[x, x^{g}\right]=1$ and $[g, x, x]=1$ for all elements $g \in G$.

(f) Every torsion ( $r s)^{\prime}$-subgroup of $G$ is a second Engel group, hence nilpotent of class less than or equal to three.

(g) Assume that $G$ has a unique minimal normal subgroup $N$. If $x^{r} \neq 1$ for some element $x \in G$, then $x^{s} \in C_{G}(N)$.

PROOF. Part (a) is clear. For (b), note that we have

$$
\left[y, x^{s}, x^{r}\right]=\left[\left(x^{-s}\right)^{y} x^{s}, x^{r}\right]=\left[\left(x^{-s}\right)^{y}, x^{r}\right]^{x^{s}}=1 .
$$

For (c), let $r^{\prime}=\lambda r$ and $s^{\prime}=\mu s$. As $G \in \mathcal{O}(r, s)$, for all $x, y \in G$ the elements $a=x^{r}$ and $b=\left(x^{s}\right)^{y}$ commute. Clearly, also the powers $a^{\lambda}=x^{r^{\prime}}$ and $b^{\mu}=\left(x^{s^{\prime}}\right)^{y}$ of $a$ and $b$ commute. This shows $G \in \mathcal{O}\left(r^{\prime}, s^{\prime}\right)$.

For (d), write $n=\lambda r$ and $n=\mu s$ for some positive integers $\lambda, \mu$. By (c), we have $G \in \mathcal{O}(n, n)$, and this by (b) implies $\left[y, x^{n}, x^{n}\right]=1$ for all $x, y \in G$.

For (e), we can find integers $\lambda, \mu$ such that $r \lambda \equiv s \mu \equiv 1 \bmod (o(x))$. As $x^{r}$ and $\left(x^{g}\right)^{s}$ commute, so do their powers $\left(x^{r}\right)^{\lambda}=x$ and $\left(\left(x^{g}\right)^{s}\right)^{\mu}=x^{g}$. Part (f) is obvious from (e).

For (g), let $R=\left\langle\left(x^{r}\right)^{G}\right\rangle$. As $x^{r} \neq 1$, we have $R \neq 1$, and so, from the hypothesis, we get $N \leq R$. By (a), we have $x^{s} \in C_{G}(R) \leq C_{G}(N)$.

As we have seen in Example 2, the class $\mathcal{O}(2,3)$ contains $\mathfrak{A A}_{2}$ and $\mathfrak{A A}_{3}$. This, in some sense, is worst possible because of the following result.

THEOREM 5. Let $G$ be a group.

(a) Let $G \in \mathcal{O}(r, s)$, and set $n=\operatorname{lcm}(r, s)$. Then $\exp (G / F(G))$ divides $n$, where $F(G)$ denotes the Hirsch-Plotkin radical of $G$.

(b) Let $r=p^{e}$ and $s=q^{f}$ be prime powers. If $G \in \mathcal{O}(r, s)$ is finite, then $G$ is soluble, and $\exp (G / F(G))$ divides $r$.

Proof. For (a), let $x \in G$. By Lemma 4(d), every two conjugates of $x^{n}$ in $G$ commute. Hence $x^{n}$ generates an Abelian normal subgroup of $G$, thus $x^{n} \in F(G)$. Thus, $G / F(G)$ is of exponent dividing $n$. For (b), note that $G / F(G)$ by (a) is of exponent dividing $r s=p^{e} q^{f}$. Hence $G$ is soluble. 
COROLLARY 6. Every group in $\mathcal{O}(1,2)$ is locally supersoluble.

Proof. Let $G \in \mathcal{O}(1,2)$. By Theorem $5, G^{2}$ is locally nilpotent. Let $H$ be a finitely generated subgroup of $G$. Then $H / H^{2}$ is finite, so that $H^{2}$ is finitely generated hence nilpotent. This implies that $H$ is supersoluble.

In view of Corollary 6 one might ask whether every group in $\mathcal{O}(1,2)$ is even metabelian. This, however, is not the case as we shall now show the following result.

EXAMPLE 7. Let $p$ be an odd prime, and let $N=\langle a, b\rangle$ be the relatively free two-generator group of exponent $p$ and class two. Then $|N|=p^{3}$, and $N$ has an automorphism $z$ with $a^{z}=a^{-1}$ and $b^{z}=b^{-1}$. Note that $z$ is of order two, and $z$ centralizes $c:=[a, b]$. Let $G=[N]\langle z\rangle$ be the canonic split extension. Then $G^{\prime}=N$ is nonabelian, so that $G$ is not metabelian.

Note that $z$ inverts $N /\langle c\rangle$, so that every subgroup of $N$ containing $c$ is normal in $G$. We claim $G \in \mathcal{O}(1,2)$. For this, let $x, y \in G$. If $x \in N$, then the above comment shows $\left\langle x^{G}\right\rangle \leq\langle x, c\rangle$, so that $\left\langle x^{G}\right\rangle$ is Abelian. Hence $\left[x,\left(x^{2}\right)^{y}\right]=1$. Next, assume $x \in G \backslash N$. Then $x=n z$ for some $n \in N$. As $z$ inverts $N /\langle c\rangle$, this yields $x^{2} \in\langle c\rangle$. As $\langle c\rangle=Z(G)$, we arrive at $\left[x,\left(x^{2}\right)^{y}\right]=1$ as claimed.

QUESTION Is every 2-group in $\mathcal{O}(1,2)$ metabelian? Is every group in $\mathcal{O}(1,2)$ centreby-metabelian?

COROLlary 8. All finite groups in $\mathcal{O}(r, s)$ are supersoluble if and only if $r \leq 2$ and $s \leq 2$.

Proof. Let $G \in \mathcal{O}(r, s)$. If $r \leq 2$ and $s \leq 2$, then by Theorem 5(a), we have that $G / F(G)$ is of exponent two, and hence $G$ is supersoluble. For the converse, assume $r \geq 3$. By Example 2, we have $\mathfrak{A} \mathfrak{A}_{r} \subseteq \mathcal{O}(r, s)$. As $\mathfrak{A A}_{r}$ contains finite groups which are nonsupersoluble (for example the wreath product $\mathbb{Z}_{p}$ wr $\mathbb{Z}_{r}$ for a prime $p$ not dividing $r$ with $p \not \equiv 1 \bmod r$ ), we have a contradiction. The result follows.

COROllary 9. All groups (finite or infinite) in $\mathcal{O}(r, s)$ are nilpotent if and only if $r=s=1$.

PROOF. Clearly, every group in $\mathcal{O}(1,1)$ is nilpotent of class $\leq 3$. For the converse, Corollary 8 implies that we have $r \leq 2$ and $s \leq 2$. It suffices to note that $S_{3} \in$ $\mathcal{O}(1,2)=\mathcal{O}(2,1)$ and $S_{3} \in \mathcal{O}(2,2)$.

In the situation of Theorem 5(a), we conjecture that $G^{n}$ is even a second Engel group. We can prove this in a special case.

Corollary 10. Let $G \in \mathcal{O}(r, s)$, and set $n=\operatorname{lcm}(r, s)$. If $G$ is torsion free, then $G^{n}$ is nilpotent of class two.

Proof. Let $x, y, z \in G^{n}$, and let $H=\langle x, y, z\rangle$. By Theorem 5(a), we know that $H$ is nilpotent Let $p$ be a prime with $p \nmid 3 n$. Then $H$ is residually a finite $p$-group. By 
Lemma 4(f), every such finite quotient is second-Engel, hence of class two (here we use $p \neq 3$ ). This shows $[x, y, z]=1$. Hence $G^{n}$ is of class two.

Note that the derived length of groups in $\mathcal{O}(r, s)$, in general is not bounded by some function of $r$ and $s$. For example, every group of exponent four is in $\mathcal{O}(1,4)$, and by [8] there are such groups of arbitrary derived length.

COROLLARY 11. There exists a function $f$ with the following property. If $G \in \mathcal{O}(r, s)$ is finite and soluble, then the Fitting length of $G$ is less than or equal to $f(r, s)$.

PROOF. By Theorem 5(a), it suffices to show that the Fitting length of a finite soluble group $H$ of exponent $r s$ is bounded. Let $K$ be a two-generator subgroup of $H$. By the solution of the restricted Burnside problem (see $[9,10]$ ), $K$ is of bounded order, thus of bounded Fitting length less than or equal to $f_{0}(r, s)$, say. By [4], also $H$ is of Fitting length less than or equal to $f_{0}(r, s)$.

The following shows that simple groups $G \in \mathcal{O}(r, s)$ have bounded structure. In particular, the element orders of $G$ can be recovered from $r$ and $s$.

COROLlary 12 (See [3]). Let $G \in \mathcal{O}(r, s)$ be a nonabelian simple group (possibly infinite). Then every element of $G$ has finite order dividing $r$ or $s$. In particular, the exponent of $G$ is finite.

Proof. Suppose the corollary is false. Then there exists $x \in G$ with $x^{r} \neq 1$ and $x^{s} \neq 1$. Let $N=\left\langle\left(x^{s}\right)^{G}\right\rangle$. By Lemma 4(a), we have $x^{r} \in C_{G}(N)$. As $x^{s} \neq 1$, we have $N \neq 1$. As $N$ is normal in $G$ and $G$ is simple, we get $N=G$, and so $1 \neq x^{r} \in C_{G}(N)=Z(G)$. Hence we have a contradiction.

COROLlary 13. For every pair $r$, s of positive integers, the class $\mathcal{O}(r, s)$ contains only finitely many nonabelian finite simple groups.

PROOF. By Corollary 12, the exponent of a simple group in $\mathcal{O}(r, s)$ is bounded by $r s$. It is well known from the classification, that there are only finitely many nonabelian finite simple groups of exponent dividing $r s$.

\section{Small values for $r$ and $s$}

We now consider finite groups in $\mathcal{O}(r, s)$ where $r$ and $s$ are particularly simple. For this, we need to consider some very specific groups that occur naturally as minimal counterexamples.

LEMMA 14 (See [1]). Let $\mathcal{K}$ be a subgroup-closed formation of finite soluble groups, and let $G$ be a finite soluble group all of whose proper subgroups and quotients are in $\mathcal{N} \mathcal{K}$, but $G \notin \mathcal{N} \mathcal{K}$. Then $G=[N] Q$ where $N=C_{G}(N)=F(G)$ is the unique minimal normal subgroup of $G$. Moreover, $Q \notin \mathcal{K}$, but every proper subgroup of $Q$ belongs to $\mathcal{K}$. 
The following result determines further properties of a group in $\mathcal{O}(r, s)$ which has the structure as in Lemma 14.

LEMMA 15. Let $G=[N] Q$ be group where $N=C_{G}(N)$ is the unique minimal normal subgroup of $G$. Assume that $G \in \mathcal{O}(r, s)$. Then we have the following results.

(a) Let $x \in G$. If $x^{r} \neq 1$, then $x^{s} \in N$.

(b) For every $x \in Q$, we have $x^{r}=1$ or $x^{s}=1$.

(c) Assume that $r$ and $s$ are coprime. Then for all $x \in G \backslash N$, we have $x^{r}=1$ or $x^{s}=1$.

Proof. (a) By Lemma 4(g), we have $x^{s} \in C_{G}(N)=N$.

(b) Assume $x^{r} \neq 1$. By (a), we have $x^{s} \in Q \cap N=1$.

(c) Let $x \in G \backslash N$. By way of contradiction, suppose $x^{r} \neq 1$ and $x^{s} \neq 1$. By Lemma 4(a), we have $x^{s} \in N$ and $x^{r} \in N$. By hypothesis, $r$ and $s$ are coprime, so there exist $\lambda, \mu \in \mathbb{Z}$ with $\lambda r+\mu s=1$. We arrive at $x=x^{\lambda r+\mu s}=\left(x^{r}\right)^{\lambda}\left(x^{s}\right)^{\mu} \in N$, against the hypothesis $x \in G \backslash N$.

COROLlary 16. Under the hypothesis of Lemma 15, let $x \in Q, x \neq 1$. If o (x) and $|N|$ are coprime then $x$ acts fixed point freely on $N$, that is, $C_{N}(x)=1$.

COROLLARY 17. Under the hypothesis of Lemma 15, assume that $r$ and s are powers of two distinct primes. Then every element of $G$ is of prime power order.

We now discuss when one of the classes $\mathcal{O}(r, s)$ is contained in another. Note that a sufficient condition is contained in Lemma 4(c): for all $r, s$ and all $\lambda, \mu$, we have $\mathcal{O}(r, s) \subseteq \mathcal{O}(\lambda r, \mu s)$. We now consider whether the converse of this might be true.

COROllary 18. Let $r$ and $s$ be positive integers. If $\mathcal{O}(r, s) \subseteq \mathcal{O}\left(r^{\prime}, s^{\prime}\right)$ for some positive integers $r^{\prime}, s^{\prime}$, then $r$ divides $r^{\prime}$ or $s^{\prime}$, and $s$ divides $r^{\prime}$ or $s^{\prime}$.

PROOF. The result is clear for $r=1$, so let $r>1$. Let $G=[N] Q$ be a Frobenius group where $Q=\langle x\rangle$ is cyclic of order $r$, and $|N|=p \equiv 1 \bmod r$ where $p$ is a prime.

By Example 3, we have $G \in \mathcal{O}(r, 1) \subseteq \mathcal{O}(r, s)$, hence the hypothesis yields $G \in$ $\mathcal{O}\left(r^{\prime}, s^{\prime}\right)$. From Lemma $15(\mathrm{~b})$, we get $x^{r^{\prime}}=1$ or $x^{s^{\prime}}=1$. Hence $r=o(x)$ divides either $r^{\prime}$ or $s^{\prime}$ as claimed. The second assertion follows from an analogous argument.

Question Suppose that $\mathcal{O}(r, s) \subseteq \mathcal{O}\left(r^{\prime}, s^{\prime}\right)$. Does it follow that $r\left|r^{\prime}, s\right| s^{\prime}$ or $r\left|s^{\prime}, s\right| r^{\prime}$ ?

We can now set out to determine properties of the class $\mathcal{O}(r, s)$ where $r$ and $s$ are 'small' in some sense. First we consider the case when $r=1$.

THEOREM 19. Let $p^{e}$ be a prime power, and let $G \in \mathcal{O}\left(1, p^{e}\right)$ be a finite group.

(a) If $p \neq 2$, then $G / F(G)$ is an Abelian $p$-group.

(b) If $p=2$, then $G / F(G)$ is a 2-group of class $\leq e$. 
ProOf. Note that by Theorem 5, we have that $\exp (G / F(G))$ divides $p^{e}$, so that $G$ is soluble. Let $\mathcal{K}$ be the class of all finite Abelian $p$-groups if $p \neq 2$ and the class of all finite 2-groups of nilpotent class $\leq e$ if $p=2$. In both cases, $\mathcal{K}$ is QSD-closed.

Let $G$ be a counterexample to (a) or (b) of least possible order. By minimality, every proper subgroup and quotient of $G$ is in $\mathcal{N} \mathcal{K}$. As $G$ is soluble, it has the form $G=[N] Q$ as described in Lemma 14.

By Lemma 15(c), every element in $G \backslash N$ has order dividing $p^{e}$. Now $N$ is an elementary Abelian $q$-group for some prime $q$, say. Clearly, $q \neq p$. Hence every element $\neq 1$ of $Q$ acts fixed point freely on $N$ (otherwise, $G \backslash N$ would contain elements of order $p q$ ), so $Q$ is a Frobenius complement. We now use the classification of Frobenius complements (see [5, p. 505]). If $p \neq 2$, then the $p$-group $Q$ is cyclic thus $G / F(G)=Q$ is Abelian against minimality. If $p=2$, the 2-group $Q$ is cyclic or generalized quaternion of exponent dividing $2^{e}$. Thus, $Q$ is nilpotent of class $\leq e$ and $Q \in \mathcal{K}$. But this is against minimality.

The following shows that the bound given in (b) of the above theorem is best possible.

EXAMPLE 20. Let $q$ be a prime with $q \equiv 1 \bmod 2^{e}$, and let $Q$ be a Sylow 2-subgroup of $\operatorname{SL}(2, q)$. Then $Q$ is a generalized quaternion group of exponent $2^{e}$. Let $N$ be the elementary Abelian group of order $q^{2}$, and let $G=[N] Q$ be the natural split extension. Then $G$ is a Frobenius group with kernel $N$ and complement $Q$. In particular, every element of $G \backslash N$ is of order dividing $2^{e}$. Example 3 yields $G \in \mathcal{O}\left(1,2^{e}\right)$, and $G / F(G)$ is of class $e$ precisely.

The next case is when $r=1$, and $s=p q$ is a product of two primes.

THEOREM 21. Let $p$ and $q$ be primes, and let $G \in \mathcal{O}(1, p q), G$ finite. Then $G$ is metanilpotent.

PROOF. For $p=q$, the result follows immediately from Theorem 5, so let $p \neq q$. Note that $G$ by Theorem 5 is soluble. Let $G$ be a counterexample of least possible order. By Lemma 14, we have $G=[N] Q$ where $N=C_{G}(N)$ is the unique minimal normal subgroup of $G$, and $Q$ is minimal nonnilpotent.

By Lemma 15(c), every element of $G \backslash N$ is of order dividing $p q$. Let $r$ be the prime dividing the order of $N$. First, assume $r \notin\{p, q\}$. As $G \backslash N$ does not contain any element of order divisible by $r$, the group $Q$ must act fixed point freely on $N$. As $\exp (Q)$ divides $p q$, the structure of Frobenius complements (see [5, p. 505]) shows that $Q$ is cyclic, hence Abelian, so that we have a contradiction. Hence $r \in\{p, q\}$. By symmetry, we may assume $r=p$. Let $P$ be a Sylow $p$-subgroup of $G$. By Lemma 15(c), every element of $P \backslash N$ is of order $p$, so $\exp (P)=p$. An appeal to Hall and Higman's Theorem B (see [6, p. 451f]) shows that $G$ is of $p$-length one. As $N=C_{G}(N)$, we have $O_{p^{\prime}}(G)=1$, so $P \unlhd G$, and $G / P$ is a $q$-group. Here, $G$ is metanilpotent, a final contradiction. 
Note that groups in $\mathcal{O}(1,2 p)$ generally need not be metabelian as can be seen from Example 7. Moreover, every faithful extension of an elementary Abelian 2-group by the nonabelian group of exponent $p \neq 2$ and order $p^{3}$ is of exponent $2 p$, hence lies in $\mathcal{O}(1,2 p)$. But this group does not have a nilpotent derived subgroup.

We now consider the case where $r$ and $s$ are prime powers.

THEOREM 22. Let $r=p_{1}^{e_{1}}$ and $s=p_{2}^{e_{2}}$ be prime powers, and let $G \in \mathcal{O}(r, s)$ be a finite group.

(a) $G / F(G)$ is supersoluble.

(b) If $p_{1} \nmid p_{2}-1$ and $p_{2} \nmid p_{1}-1$, then $G$ is metanilpotent.

(c) If $e_{1}=e_{2}=1$ and $p_{1} \neq p_{2}$, then $G / F(G)$ is Abelian.

Proof. If $p_{1}=p_{2}$, then by Theorem $5(\mathrm{a})$, the quotient $G / F(G)$ is a $p_{1}$-group, and so (a) and (b) hold. We thus may assume $p_{1} \neq p_{2}$.

(a) By Theorem 5(b), the group $G$ is soluble. Let $G$ be a counterexample of least possible order. By Lemma 14, we have that $G=[N] Q$ where $N=F(G)$ is the unique minimal normal subgroup of $G$. Moreover, $N=C_{G}(N)$, and every proper subgroup of $Q$ is supersoluble, but $Q$ is not supersoluble. A result of Doerk (see [5, p. 721]) yields that $Q=[A] B$ where $A$ is a noncyclic normal Sylow subgroup of $Q$, and $B$ acts irreducibly on $A / \Phi(A)$ which is a noncyclic chief factor of $G$.

As $Q$ acts faithfully and irreducibly on $N$, we have $(|N|,|A|)=1$. By Lemma 15 (c), every element in $G \backslash N$ is of prime power order. Hence $C_{N}(a)=1$ for all $a \in A, a \neq 1$. By the classification of Frobenius complements (see [5, p. 505]) $A$ is either cyclic or generalized quaternion. However, $A / \Phi(A)$ is noncyclic, so that the first case does not occur. Let $A$ be generalized quaternion. As $B$ acts irreducibly on $A / \Phi(A)$, we see that $B$ induces on $A$ an automorphism of order three which clearly centralizes the involution in $A$. In particular, $Q$ contains elements of order six. But all elements of $G \backslash N$ are of prime power order, so we have a contradiction.

(b) Let $G$ be a counterexample of least possible order. As in (a), we have $G=[N] Q$ where $N=C_{G}(N)$, and every proper subgroup of $Q$ is nilpotent. By (a), the complement $Q$ is supersoluble.

By the structure of minimal nonnilpotent groups (see [5, p. 281]), we get $Q=[A] B$ where $A$ and $B$ are cyclic. By Lemma 15(c), every element in $G \backslash N$ is of order dividing $p_{1}^{e_{1}}$ or $p_{2}^{e_{2}}$. So $\pi(Q)=\left\{p_{1}, p_{2}\right\}$. As $Q$ is nonnilpotent, this implies $p_{1} \mid p_{2}-1$ or $p_{2} \mid p_{1}-1$ against the hypothesis.

(c) Let $G$ be a counterexample of least possible order. As in (a), we have $G=[N] Q$ where $N=C_{G}(N)$, and every proper subgroup of $Q$ is Abelian. By (a), we know that $Q$ is supersoluble.

First, assume that $Q$ is nilpotent, thus a $p$-group for some prime $p$. Вy Lemma 15 (c), every element in $G \backslash N$ is of order $p_{1}$ or $p_{2}$, so $\exp (Q)=p \in\left\{p_{1}, p_{2}\right\}$, and $Q$ does not contain any subgroup $\cong \mathbb{Z}_{p} \times \mathbb{Z}_{p}$. This implies that $Q \cong \mathbb{Z}_{p}$ is Abelian, so we have a contradiction.

We may assume $p_{1}>p_{2}$. As $\exp (Q)$ divides $p_{1} p_{2}$, the minimal nonabelian group $Q$ is isomorphic to the nonabelian split extension of $\mathbb{Z}_{p_{1}}$ by $\mathbb{Z}_{p_{2}}$. Assume that $N$ 
is a $q$-group for the prime $q$. If $q \notin\left\{p_{1}, p_{2}\right\}$, then $Q$ would be a Frobenius complement of $G$, but this is against the well-known structure of these. As $Q$ acts faithfully and irreducibly on $N$, we have $q \neq p_{1}$. Hence $N$ is a $p_{2}$-group. Now consider a Sylow $p_{2}$-subgroup $P$ of $G$. By Lemma 15(c), every element of $G \backslash N$ is of order $p_{1}$ or $p_{2}$, so every element of $P \backslash N$ is of order $p_{2}$. Hence $\exp (P)=p_{2}$. By Hall and Higman's Theorem B (see [6, p. 451f]), $G$ is of $p_{2}$-length one. As $O_{p_{1}}(G)=1$, the group $G$ is $p_{2}$-closed, so we have a contradiction.

\section{REMARK 23.}

(a) Part (c) of Theorem 22 is no longer true in the case when $p=q$. Indeed, let $G$ be an extension of an Abelian normal subgroup by a group of exponent $p$. Then $G \in \mathcal{O}(p, p)$, but $G / F(G)$ is not necessarily Abelian if $p>2$. Moreover, for large $p$, there exist infinite simple groups of exponent $p$, and so, in general, $G$ need not even be soluble. Here, one cannot dispose with that hypothesis that $G$ is finite.

(b) Part (c) of Theorem 22 is no longer true for arbitrary $e_{1}, e_{2}$. Indeed, the symmetric group $G=S_{4}$ has elements of orders one, two, three and four. Hence we have $G \in \mathcal{O}(3,4)$. But $G / F(G) \cong S_{3}$ is nonabelian.

QUESTION Assume that all finite groups in $\mathcal{O}(r, s)$ are metanilpotent. What can be said about $r$ and $s$ ?

\section{Acknowledgement}

The material has been presented at the Conference 'Ischia Group Theory 2008' on 2nd-4th April, 2008. The author is grateful to the organizers for their most generous support.

\section{References}

[1] R. Brandl, 'Zur Theorie der untergruppenabgeschlossenen Formationen: Endliche Varietäten', J. Algebra 73 (1981), 1-22.

[2] R. Brandl and L. C. Kappe, 'On n-Bell groups', Comm. Algebra 17 (1989), 787-807.

[3] R. M. Bryant and M. B. Powell, 'Two-variable laws for PSL (2, 5)', J. Aust. Math. Soc. 10 (1969), 499-502.

[4] R. Carter, B. Fischer and T. O. Hawkes, 'Extreme classes of finite groups', J. Algebra 9 (1968), 285-313.

[5] B. Huppert, Endliche Gruppen, Vol. I (Springer, Berlin, 1967).

[6] B. Huppert and N. Blackburn, Finite Groups, Vol. II (Springer, Berlin, 1982).

[7] L. C. Kappe, 'On n-Levi groups', Arch. Math. 47 (1986), 198-210.

[8] M. Vaughan-Lee, 'Derived lengths of Burnside groups of exponent 4', Q. J. Math. (Oxford) II. Ser. 30 (1979), 495-504.

[9] E. I. Zelmanov, 'Solution of the restricted Burnside problem for groups of odd order', Izv. Akad. Nauk SSSR Ser. Mat. 54(1) (1990), 42-59.

[10] - 'Solution of the restricted Burnside problem for 2-groups', Mat. Sb. 182(4) (1991), $568-592$.

ROLF BRANDL, Mathematisches Institut, Am Hubland 12, 97074 Würzburg, Germany 